\title{
Análise de elementos finitos de um dispositivo de dinamização controlada para fixação circular externa
}

Finite Element Analysis of a Controlled Dynamization Device for External Circular Fixation

\author{
Fernando Ferraz Faria1 ${ }^{10}$ Carlos Eduardo Miers Gruhl ${ }^{10}$ Rafaela Rebonato Ferro ${ }^{2}$ (1) \\ Rodrigo Nunes Rached ${ }^{3 \odot}$ Jamil Faissal Soni ${ }^{4 \odot}$ Paula Trevilatto ${ }^{3 \odot}$ \\ ${ }^{1}$ Escola de Ciências da Vida, Departamento de Ciências da Saúde, Pontifícia \\ Universidade Católica do Paraná (PUCPR), Curitiba, Paraná, Brazil \\ Endereço para correspondência Paula Trevilatto, PhD, Pontifícia \\ 2 Departamento de Ortopedia e Traumatologia, Hospital Universitário \\ Cajuru, Pontifícia Universidade Católica do Paraná (PUCPR), Curitiba, \\ Universidade Católica do Paraná, Curitiba, PR, Brasil \\ Paraná, Brazil \\ 3 Programa de Pós-Graduação em Odontologia, Faculdade de Ciências \\ da Vida, Pontifícia Universidade Católica do Paraná (PUCPR), \\ Curitiba, Paraná, Brazil \\ ${ }^{4}$ Programa de Pós-Graduação em Medicina, Pontifícia Universidade \\ Católica do Paraná (PUCPR), Curitiba, Paraná, Brazil \\ (e-mail: paulatrevilatto@pucpr.br). \\ Rev Bras Ortop 2021;56(1):36-41.
}

\begin{abstract}
Resumo
Palavras-chave

- fraturas ósseas

- fixadores externos

- consolidação da fratura

- dinamização

Objetivo Construir um protótipo virtual de um dispositivo de fixação circular externa para fraturas em ossos longos com dinamização controlada a partir de dois materiais diferentes e prever seu comportamento mecânico por meio da análise de elementos finitos AEF).

Método Modelos tridimensionais compostos de duas peças metálicas unidas por uma junta deslizante em rabo de andorinha e um amortecedor de silicone de alta densidade foram criados em um software. Análises de elementos finitos distintas foram simuladas considerando dois materiais (aço inoxidável ou titânio), modos (bloqueado ou dinamizado) e condições de carregamento (estático/pontual ou dinâmico/0,5 segundo) diferentes com carga axial uniforme de $150 \mathrm{~kg}$ na porção superior do dispositivo.

Resultados $\mathrm{O}$ modelo de elementos finitos (EFs) apresentou 81.872 nós e 45.922 elementos. Com aço inoxidável, o pico de tensão máxima (140,98 MPa) foi alcançado com o dispositivo bloqueado e sob carga estática, enquanto o maior deslocamento $\left(2.415 \times 10^{-3} \mathrm{~mm}\right)$ foi obtido com o dispositivo bloqueado e sob carga dinâmica. Com titânio, o pico de tensão máxima (141,45 MPa) ocorreu com o dispositivo bloqueado e sob carga estática, enquanto o maior deslocamento $\left(3.975 \times 10^{-3} \mathrm{~mm}\right)$ foi observado com o dispositivo bloqueado e sob carga dinâmica.

Conclusão O protótipo do dispositivo desempenhou o papel de suporte de tensão com deformação aceitável nos dois modos, bloqueado ou dinamizado, e pode ser fabricado com aço inoxidável ou titânio.
\end{abstract}

recebido

31 de Janeiro de 2020

aceito

17 de Setembro de 2020
DOI https://doi.org/

$10.1055 / \mathrm{s}-0040-1721368$

ISSN 0102-3616.
(C) 2021. Sociedade Brasileira de Ortopedia e Traumatologia. All rights reserved.

This is an open access article published by Thieme under the terms of the Creative Commons Attribution-NonDerivative-NonCommercial-License, permitting copying and reproduction so long as the original work is given appropriate credit. Contents may not be used for commercial purposes, or adapted, remixed, transformed or built upon. (https://creativecommons.org/ licenses/by-nc-nd/4.0/)

Thieme Revinter Publicações Ltda., Rua do Matoso 170, Rio de Janeiro, RJ, CEP 20270-135, Brazil 
Abstract

\section{Keywords \\ - fractures, bone \\ - external fixators \\ - fracture healing \\ - dynamization}

Objective to virtually prototype a device for external circular fixation of long bone fractures with controlled dynamization made of two different materials and predict their mechanical behavior by using finite element method analysis (FEA).

Method A software was used for 3D modeling two metal parts closely attached by a sliding dovetail joint and a high-density silicone damper. Distinctive FEAs were simulated by considering two different materials (stainless steel or titanium), modes (locked or dynamized) and loading conditions (static/point or dynamic/0.5 sec) with uniform $150 \mathrm{~kg}$ axial load on top of the device.

Results The finite elements (FEs) model presented 81,872 nodes and 45,922 elements. Considering stainless steel, the maximum stress peak (140.98 MPa) was reached with the device locked under static loading, while the greatest displacement $\left(2,415 \times 10^{-3} \mathrm{~mm}\right)$ was observed with the device locked and under dynamic loading. Regarding titanium, the device presented the maximum stress peak (141.45 MPa) under static loading and with the device locked, while the greatest displacement $\left(3,975 \times 10^{-3} \mathrm{~mm}\right)$ was found with the device locked and under dynamic loading. Conclusion The prototyped device played the role of stress support with acceptable deformation in both locked or dynamized modes and may be fabricated with both stainless steel and titanium.

\section{Introdução}

O sucesso da consolidação óssea biológica parece dependente de um ambiente mecânico favorável e, segundo a lei de Wolff e a teoria de tensão (strain) de Perren, vários sistemas de osteossíntese podem ser usados para promover a estabilização adequada e os diversos tipos de diferenciação celular no sítio da fratura. ${ }^{1,2}$ A relativa estabilidade indicada para fraturas diafisárias ou cominutivas extra-articulares permite um grau controlado de mobilidade no sítio da fratura e a formação de calo ósseo exuberante, que caracteriza a ossificação indireta ou endocondral. Para prevenção da formação de calosidades ósseas volumosas em fraturas articulares, recomenda-se a ossificação direta ou intramembranosa após a fixação absoluta com maior rigidez. ${ }^{3}$

A chamada "dinamização" se refere ao uso de dispositivos de fixação externa para otimização do ambiente mecânico de osteossíntese. A fixação percutânea é um método rápido, de baixo custo e com mínima perda de sangue, que utiliza fixadores externos para estabilização de fraturas complexas com acometimento de tecidos moles ou mesmo correção progressiva de deformidades por meio da estabilização com sustentação de peso e mobilização de grandes articulações. ${ }^{4,5}$ Além disso, a formação de calo ósseo aumenta de forma significativa devido ao movimento interfragmentar, principalmente na faixa ideal de $0,5 \mathrm{~mm}$ para a aceleração da cicatrização tardia de fraturas diafisárias. ${ }^{6}$ A teoria de biocompressão, considerada tão essencial quanto os fatores osteogênicos na consolidação de fraturas ósseas, foi comprovada pelo achado de calos ósseos em pacientes com fratura de tíbia submetidos ao tratamento com fixador externo para proporcionar boa estabilidade e compressão local. ${ }^{7}$ Da mesma maneira, outros autores sugeriram o uso de fixadores externos unilaterais para promoção de dinamização controlada nas fraturas expostas e casos com retardos de consolidação. ${ }^{8,9}$ O uso de fixação externa flexível para cicatrização de fraturas em ossos longos é preconizado desde 1986 devido à maior mobilidade e formação de calo ósseo. O primeiro modelo foi utilizado por Lambotte em 1902 e modificado por Anderson e Hoffman em 1938; o clássico fixador em anel desenvolvido por Ilizarov em 1952 continua a ser uma boa opção por sua versatilidade, capacidade adaptativa, baixo custo e capacidade de aplicação de forças neutras, de compressão ou distração sobre os ossos..$^{10-12}$

A análise de elementos finitos (AEF) é muito útil devido à dificuldade de conduzir pesquisas clínicas sobre as forças necessárias para estimular a consolidação óssea. ${ }^{13}$ Este método tem sido bastante usado em medicina e ortopedia por dar uma visão geral abrangente da dissolução de vetores em estruturas debilitadas, permitir a detecção precisa de falhas e evitar custos desnecessários em casos em que a falha só seria identificada após o projeto estrutural ou fabricação. ${ }^{14}$ Além disso, o tempo transcorrido entre o primeiro projeto conceitual e a produção é reduzido, uma vez que não há necessidade de fabricação de um grande número de espécimes experimentais. A AEF gera informações que são muito difíceis de obter em condições laboratoriais, como a distribuição da tensão prevista e a resistência do material, que são de grande importância na avaliação da resistência à fadiga. ${ }^{15,16}$

A dinamização deve acelerar a consolidação dos ossos longos e melhorar a reabilitação e a recuperação dos pacientes, mas ainda não é proporcionada pelos atuais fixadores circulares externos. Assim, este estudo teve como objetivo construir um protótipo virtual de um dispositivo capaz de conectar dois anéis de um conjunto circular externo padrão e promover a dinamização. Este protótipo foi simulado com dois materiais diferentes e seu comportamento mecânico foi previsto por meio de FEA. 

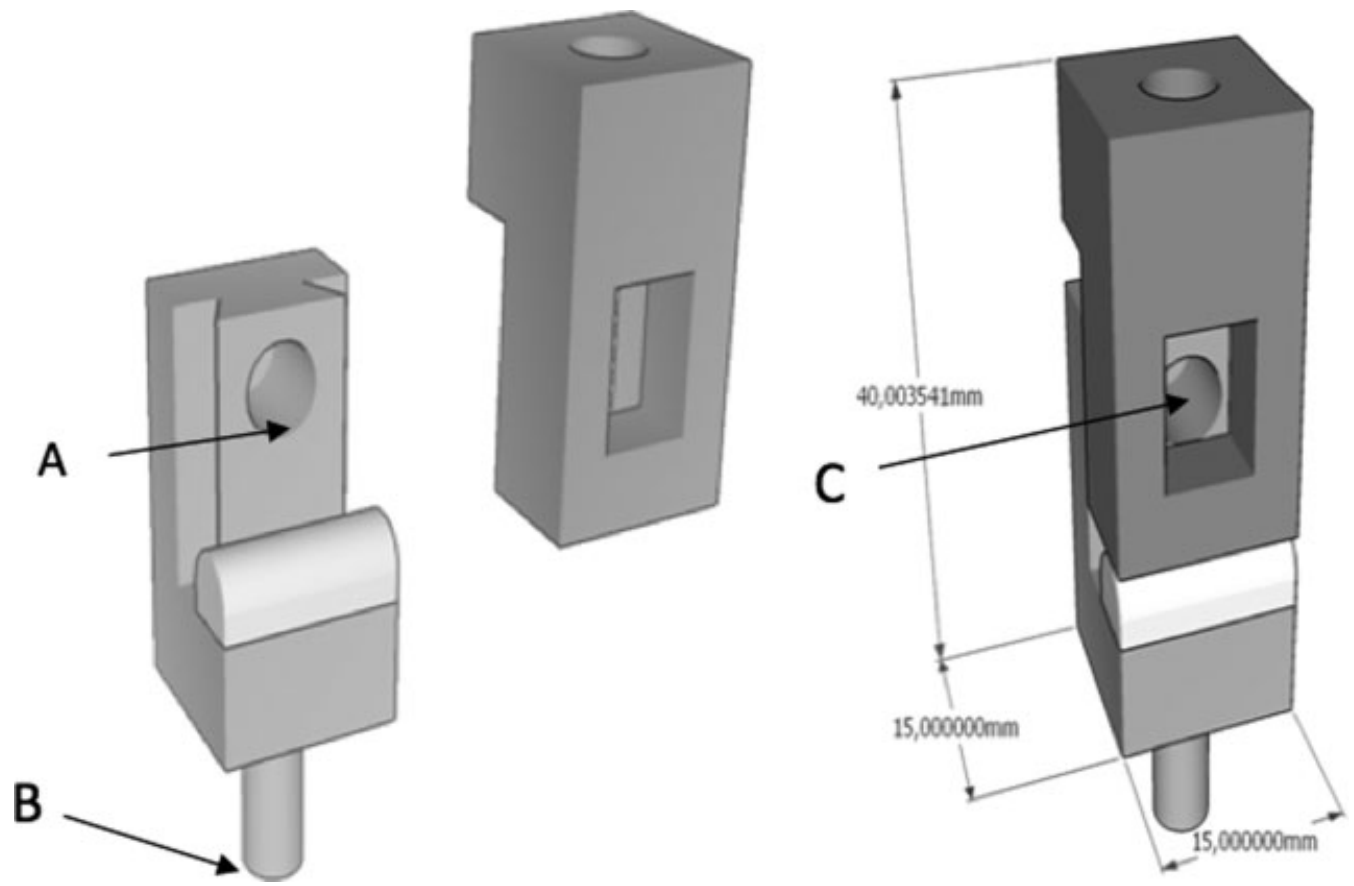

Fig. 1 Modelagem geométrica. (A) Deslizamento do rabo de andorinha. (B) Haste roscada. (C) Orifício do parafuso.

\section{Método}

\section{Modelamento geométrico}

Um software de design tridimensional (Google SketchUp 2017, Goggle LLC, Menlo Park, CA, Estados Unidos) foi utilizado para modelamento de um dispositivo (patente BR 1020170182274 registrada no Instituto Nacional da Propriedade Industrial, INPI) composto de duas partes metálicas unidas por uma junta deslizante em rabo de andorinha e um amortecedor de silicone de alta densidade (-Fig. 1). A junta em rabo de andorinha evita a separação das peças durante os movimentos angulares e de torção, enquanto o amortecedor de silicone suaviza e controla o deslocamento axial.

O dispositivo foi projetado para operação em modo bloqueado, em que as duas partes aparafusadas formam um bloco único e totalmente rígido; entretanto, nos casos em que a dinamização (modo dinâmico) é desejada, o médico afrouxa o parafuso para permitir a movimentação deslizante entre as peças e a transferência da carga para o osso. Há uma haste rosqueada na parte inferior do dispositivo. $O$ orifício na parte superior permite sua versátil conexão a um fixador externo. Além disso, o dispositivo tem aplicação universal em equipamentos de diferentes fabricantes devido à padronização de furos, parafusos e dimensões da haste.

\section{Geração de malha}

Após a modelagem geométrica, um modelo de elementos finitos (EFs) foi gerado com um software específico (Ansys r14.5, Ansys Inc., Canonsburg, PA, Estados Unidos) (-Fig. 2). O comportamento biomecânico do dispositivo foi simulado considerando as propriedades físicas de dois materiais diferentes indicados pela American Society for Testing and Materials (ASTM) para aplicações biomédicas (aço inoxidável F138/18-cromo 14-níquel 2,5-molibdênio ou titânio

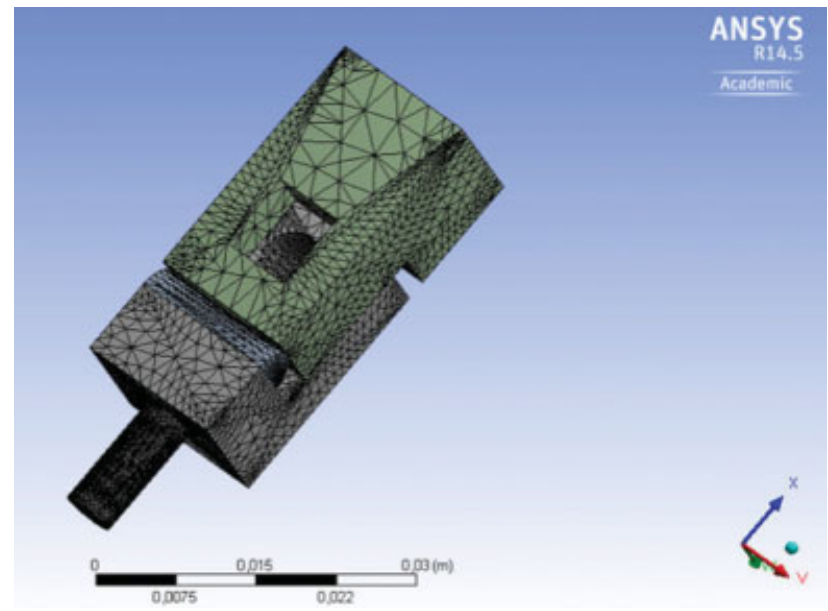

Fig. 2 Modelo de elementos finitos com 81.872 nós e 45.922 elementos.

F1295/Ti6Al7Nb) para prever sua viabilidade para posterior usinagem (-Tabela $\mathbf{1}$ ).

\section{Simulação virtual}

Durante a aplicação de uma carga de $500 \mathrm{~N}$ que, de acordo com a Food and Drug Administration (FDA) dos Estados Unidos, corresponde a um peso corpóreo humano de 150 $\mathrm{Kg}$, o modelo foi submetido à análise estrutural de deslocamento intrínseco, deformação e sítio suscetível à fadiga. Essa carga se justifica pelo uso simultâneo de pelo menos três dispositivos em uma situação de vida real. A carga foi aplicada em direção ao centro do dispositivo, em sua superfície superior, com inclinação constante de $90^{\circ}$.

Para simulação dos estágios de tratamento bloqueado (sem movimento no sítio da fratura) e dinâmico (com 
Tabela 1 Propriedades físicas dos materiais

\begin{tabular}{|l|l|l|l|l|}
\hline Material & $\begin{array}{l}\text { Módulo de } \\
\text { elasticidade (MPa) }\end{array}$ & $\begin{array}{l}\text { Coeficiente } \\
\text { de Poisson }\end{array}$ & $\begin{array}{l}\text { Força máxima de } \\
\text { compressão (MPa) }\end{array}$ & $\begin{array}{l}\text { Força máxima de } \\
\text { tensão (MPa) }\end{array}$ \\
\hline Aço inoxidável & 187.500 & 0,33 & 800 & 800 \\
\hline Titânio & 113.800 & 0,34 & 950 & 950 \\
\hline Borracha de silicone & 0.515 & 0,4 & 0,0552 & 0,0552 \\
\hline
\end{tabular}

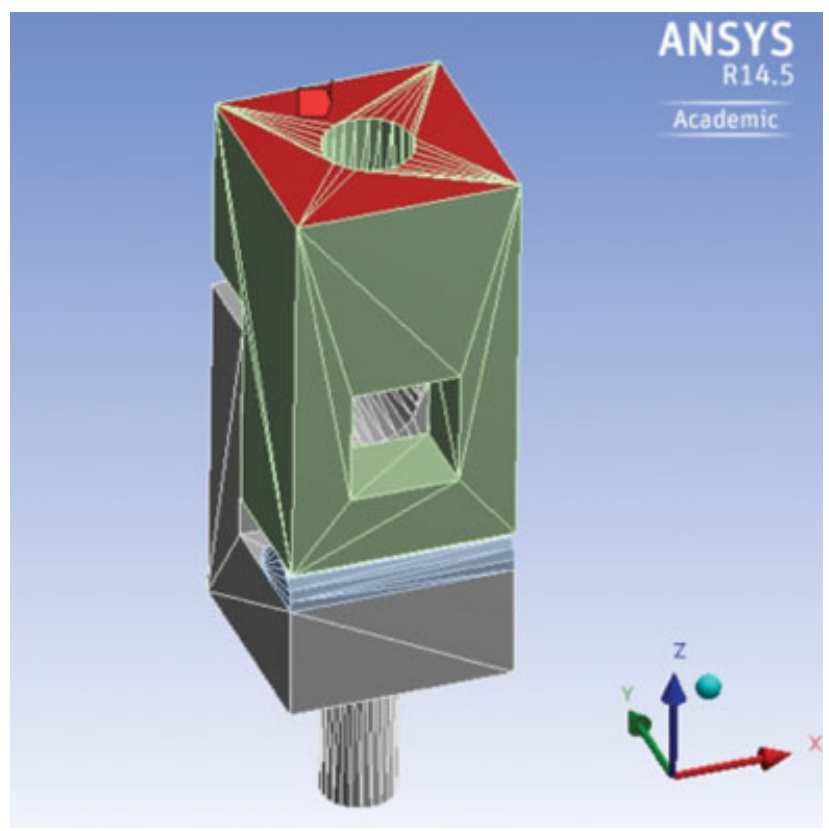

Fig. 3 Aplicação de uma carga de $150 \mathrm{~kg}$ à área vermelha.

transferência de carga para o osso e movimentação controlada), o dispositivo foi testado com as duas partes completamente fixadas uma à outra e, depois, com a possibilidade de deslocamento axial. Nos dois modos, a variável analisada após o carregamento foi o deslocamento entre as partes do dispositivo e os sítios de deformação.

A translação entre as peças foi calculada após a aplicação de forças axiais com limite de $2 \mathrm{~mm}$ de deslocamento aceitável. Os limiares de deformação sobre as forças axiais foram de $1 \mathrm{~mm}$ para o modo bloqueado e entre 1 a $2 \mathrm{~mm}$ de compressão para o modo dinâmico, com restabelecimento da forma original após a retirada da carga. $^{17}$

Considerando os materiais de construção e os modos, duas análises distintas de FE foram conduzidas para carregamento estático (pontual) ou dinâmico (0,5 segundo) com carga axial uniforme de $150 \mathrm{~kg}$ no topo do dispositivo para simulação de movimento ou posição ortostática, respectivamente (-Fig. 3). O material do dispositivo (aço inoxidável ou titânio), o modo (bloqueado ou dinamizado) e a condição de carregamento (estático ou dinâmico) foram tratados como variáveis independentes, enquanto a resistência e o deslocamento dos componentes foram analisados como variáveis dependentes.
Tabela 2 Resultados do dispositivo de aço inoxidável

\begin{tabular}{|l|l|l|l|l|}
\hline $\begin{array}{l}\text { Modo do } \\
\text { dispositivo }\end{array}$ & \multicolumn{2}{|l|}{ Bloqueado } & \multicolumn{2}{l|}{ Dinamizado } \\
\hline Carga & Estática & Dinâmica & Estática & Dinâmica \\
\hline $\begin{array}{l}\text { Tensão } \\
\text { máxima (MPa) }\end{array}$ & 140,98 & 80.637 & 9,2798 & 9,0956 \\
\hline $\begin{array}{l}\text { Deslocamento } \\
\text { máximo (mm) }\end{array}$ & $2,35 \times$ & $2,41 \times$ & $2,60 \times$ & $2,55 \times$ \\
$10^{-3}$ & $10^{-3}$ & $10^{-4}$ & $10^{-4}$ \\
\hline
\end{tabular}

Tabela 3 Resultados do dispositivo de titânio

\begin{tabular}{|l|l|l|l|l|}
\hline $\begin{array}{l}\text { Modo do } \\
\text { dispositivo }\end{array}$ & \multicolumn{2}{|l|}{ Bloqueado } & \multicolumn{2}{l|}{ Dinamizado } \\
\hline Carga & Estática & Dinâmica & Carga & Estática \\
\hline $\begin{array}{l}\text { Tensão } \\
\text { máxima (MPa) }\end{array}$ & 141,45 & 80,73 & 9,2015 & 9,0189 \\
\hline $\begin{array}{l}\text { Deslocamento } \\
\text { máximo (mm) }\end{array}$ & $\begin{array}{l}3,86 \times \\
10^{-3}\end{array}$ & $\begin{array}{l}3,97 \times \\
10^{-3}\end{array}$ & $\begin{array}{l}4,25 \times \\
10^{-4}\end{array}$ & $\begin{array}{l}4,17 \times \\
10^{-4}\end{array}$ \\
\hline
\end{tabular}

\section{Resultados}

Em conformidade ao desenvolvimento sequencial de modelagem geométrica, malha, modelagem constitutiva, condições de contorno e condições de carregamento, nosso modelo de EFs apresentou um total de 81.872 nós e 45.922 elementos. Os resultados da $\mathrm{AEF}$ em relação à tensão e deslocamento máximos obtidos com cada material, modo e condição de carregamento são descritos nas - Tabelas 2 e $\mathbf{3}$, enquanto as distribuições de tensão são mostradas na - Figura 4.

Na simulação do dispositivo em aço inoxidável, a tensão máxima não atingiu um terço da propriedade mecânica relatada, o que representa confiabilidade contra falha por fadiga quando o dispositivo bloqueado é submetido à carga estática máxima (pico de tensão máxima de 140,98 MPa na área proximal da ranhura em rabo de andorinha); as demais simulações geraram valores menores, e o menor pico de estresse, de 9,0956 MPa, foi obtido com o dispositivo dinamizado sob carga dinâmica. Além disso, o maior deslocamento do dispositivo de aço inoxidável foi observado sob carga dinâmica e em modo bloqueado $\left(2,415 \times 10^{-3} \mathrm{~mm}\right)$; entretanto, esse valor ficou bem abaixo do limite pré-estabelecido de $1 \mathrm{~mm}$. 0 deslocamento foi ainda menor ao ativar a dinamização e o menor valor foi observado em carga estática $\left(2,60 \times 10^{-4} \mathrm{~mm}\right)$ e deslocamento uniforme do 

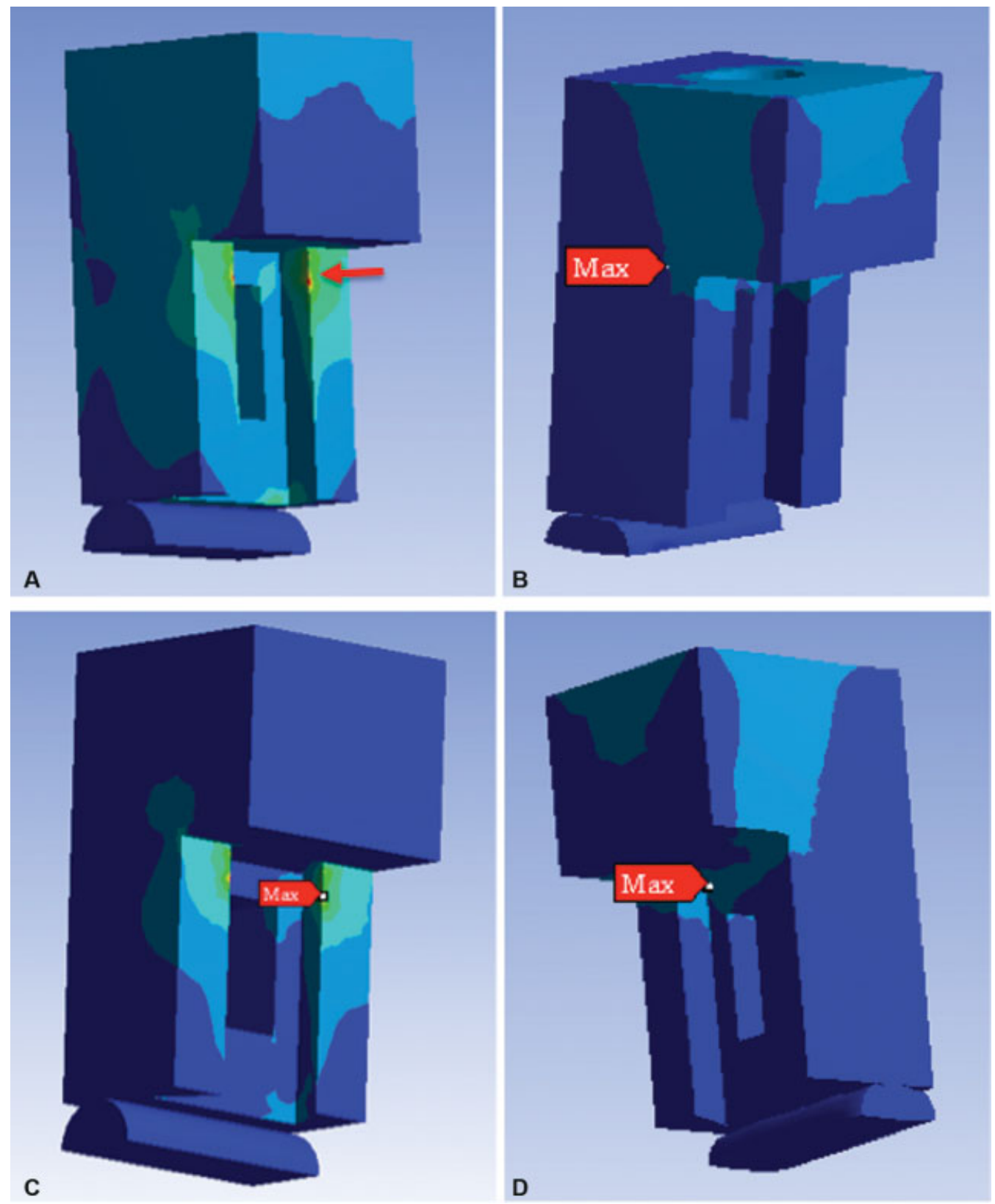

Fig. 4 Análise de elementos finitos (AEF) da distribuição de tensões sob carga dinâmica do (A) dispositivo de aço inoxidável bloqueado, (B) dispositivo de aço inoxidável dinamizado, (C) dispositivo de titânio bloqueado e (D) dispositivo de titânio dinamizado. Uma parte do dispositivo foi retirada intencionalmente da imagem para permitir a visualização dos sítios de picos de tensões máximas (indicados pelas setas vermelhas).

rabo de andorinha; a confiabilidade de promoção do deslocamento axial controlado sem interferência da deformação do dispositivo variou de 1,3477 a 2,3562 da margem de segurança.

Na simulação do dispositivo produzido em titânio, a tensão máxima observada foi 6,7 vezes menor que o limite de propriedade do material; o maior valor (141,45 MPa) foi obtido com o dispositivo bloqueado sob carga estática (pico de tensão máxima na área proximal ao rabo de andorinha) e o menor valor foi observado com o dispositivo dinamizado sob carga dinâmica $(9,0189 \mathrm{MPa})$. 0 dispositivo bloqueado sob carga dinâmica apresentou o maior deslocamento $\left(3.975 \times 10^{-3} \mathrm{~mm}\right)$ na área proximal do rabo de andorinha com margem de segurança de 10,9 , enquanto o dispositivo dinamizado sob carga estática teve o menor deslocamento $\left(4.256 \times 10^{-4} \mathrm{~mm}\right)$, e o pico de tensão máxima estava na área proximal do rabo de andorinha. Considerando que esses valores ficaram abaixo do limiar pré-estabelecido de $1 \mathrm{~mm}$, a eficácia do dispositivo em suportar cargas axiais sem interferência de deformação ou mobilidade foi demonstrada.

\section{Discussão}

Este estudo teve como objetivo desenvolver um dispositivo eficaz, mais acessível e de baixo custo para dinamização 
controlada para melhorar o uso de um fixador circular externo bastante conhecido, semelhante ao introduzido por Ilizarov. ${ }^{10} \mathrm{O}$ dispositivo para fixação circular externa foi submetido a simulações com materiais de diferentes propriedades físicas amplamente utilizados para osteossíntese: aço inoxidável altamente rígido e titânio com alta resistência à tensão e compressão. ${ }^{18}$

Em relação às diferentes cargas durante o movimento ou posição ortostática, nossos achados reforçam a confiabilidade do protótipo no controle da compressão e do deslocamento axial. As simulações do dispositivo em aço inoxidável mostraram, de forma consistente, maior deslocamento máximo em comparação ao titânio; no entanto, esses valores sempre estiveram bem abaixo do limite pré-estabelecido, assegurando a eficácia e segurança em cargas axiais realistas de até $500 \mathrm{~N} .^{19}$ A tensão máxima variou de acordo com a dinamização do dispositivo; os maiores valores foram observados com o dispositivo de titânio bloqueado tanto no carregamento estático quanto no dinâmico. Por outro lado, o dispositivo de aço inoxidável dinamizado apresentou maior tensão máxima nas duas condições de carregamento (estático e dinâmico), embora esses valores fossem inferiores a um terço da propriedade do material. ${ }^{18}$ Portanto, os dois materiais foram considerados igualmente seguros em termos de resistência e sobrecarga. Além da possibilidade de montagem com fixador circular externo sem alteração de funcionalidade inicial, o dispositivo dinamizado controlado modifica a distribuição de cargas durante o período desejado sem a necessidade de novas cirurgias.

Embora os resultados da AEF apoiem o uso deste dispositivo para a consolidação de fraturas ósseas, nossos dados devem ser interpretados com cautela e estudos clínicos randomizados controlados são definitivamente necessários para relacionar esses achados à função clínica.

\section{Conclusão}

Pode-se concluir que o protótipo desempenhou a função de suporte de tensões sem deformação em modo bloqueado ou dinamizado e pode ser fabricado tanto em aço inoxidável quanto em titânio.

Conflito de interesses

Os autores declaram não haver conflito de interesses.

\section{Referências}

1 Wolff J. Das Gesetz der Transformation der Knochen. Berlin: Hirschwald; 1892
2 Perren S, Boitzy A. Cellular differentiation and bone biomechanics during the consolidation of a fracture. Clin Anat 1978;1:13-28

3 Glatt V, Matthys R. Adjustable stiffness, external fixator for the rat femur osteotomy and segmental bone defect models. J Vis Exp 2014;9(92):e51558

4 Compton J, Fragomen A, Rozbruch SR. Skeletal Repair in Distraction Osteogenesis: Mechanisms and Enhancements. JBJS Rev 2015;3(08):pii: 01874474-201508000-00002

5 Henderson DJ, Rushbrook JL, Stewart TD, Harwood PJ. What Are the Biomechanical Effects of Half-pin and Fine-wire Configurations on Fracture Site Movement in Circular Frames? Clin Orthop Relat Res 2016;474(04):1041-1049

6 Frost HM. Bone "mass" and the "mechanostat": a proposal. Anat Rec 1987;219(01):1-9

7 Lazo-Zbikowski J, Aguilar F, Mozo F, Gonzalez-Buendia R, Lazo JM. Biocompression external fixation. Sliding external osteosynthesis. Clin Orthop Relat Res 1986;(206):169-184

8 Marsh JL, Nepola JV, Wuest TK, Osteen D, Cox K, Oppenheim W. Unilateral external fixation until healing with the dynamic axial fixator for severe open tibial fractures. J Orthop Trauma 1991;5 (03):341-348

9 Barquet A, Massaferro J, Dubra A, Milans C, Castiglioni O. The dynamic ASIF-BM tubular external fixator in the treatment of open fractures of the shaft of the tibia. Injury 1992;23(07):461-466

10 Foxworthy M, Pringle RM. Dynamization timing and its effect on bone healing when using the Orthofix Dynamic Axial Fixator. Injury 1995;26(02):117-119

11 Moss DP, Tejwani NC. Biomechanics of external fixation: a review of the literature. Bull NYU Hosp Jt Dis 2007;65(04):294-299

12 Fragomen AT, Rozbruch SR. The mechanics of external fixation. HSS J 2007;3(01):13-29

13 Roseiro LM, Neto MA, Amaro A, Leal RP, Samarra MC. External fixator configurations in tibia fractures: 1D optimization and 3D analysis comparison. Comput Methods Programs Biomed 2014; 113(01):360-370

14 Lotti RS, Machado AW, Mazzieiro ET, Landre Júnior J. Scientific application of finite elemento method. Rev Dent Press Ortodon Ortop Facial 2006;11:35-43

15 Easley SK, Pal S, Tomaszewski PR, Petrella AJ, Rullkoetter PJ, Laz PJ. Finite element-based probabilistic analysis tool for orthopaedic applications. Comput Methods Programs Biomed 2007;85(01):32-40

16 Kluess D, Souffrant R, Mittelmeier W, Wree A, Schmitz KP, Bader R. A convenient approach for finite-element-analyses of orthopaedic implants in bone contact: modeling and experimental validation. Comput Methods Programs Biomed 2009;95(01):23-30

17 Burgers PT, Van Riel MP, Vogels LM, Stam R, Patka P, Van Lieshout EM. Rigidity of unilateral external fixators-a biomechanical study. Injury 2011;42(12):1449-1454

18 Gao Y, Jin Z, Wang L, Wang M. Finite element analysis of sliding distance and contact mechanics of hip implant under dynamic walking conditions. Proc Inst Mech Eng H 2015;229(06):469-474

19 Pan M, Chai L, Xue F, Ding L, Tang G, Lv B. Comparisons of external fixator combined with limited internal fixation and open reduction and internal fixation for Sanders type 2 calcaneal fractures: Finite element analysis and clinical outcome. Bone Joint Res 2017; 6(07):433-438 